1991-03-01

\title{
Theory of an atomic beam splitter based on velocity-tuned resonances
}

\author{
Scott Glasgow \\ Glasgow@mathematics.byu.edu \\ P. Meystre \\ M. Wilkens \\ E. M. Wright
}

Follow this and additional works at: https://scholarsarchive.byu.edu/facpub

Part of the Astrophysics and Astronomy Commons, and the Physics Commons

Original Publication Citation

Physical Review A, vol 43, no 5, pp 2455-2463.

\section{BYU ScholarsArchive Citation}

Glasgow, Scott; Meystre, P.; Wilkens, M.; and Wright, E. M., "Theory of an atomic beam splitter based on velocity-tuned resonances" (1991). Faculty Publications. 722.

https://scholarsarchive.byu.edu/facpub/722

This Peer-Reviewed Article is brought to you for free and open access by BYU ScholarsArchive. It has been accepted for inclusion in Faculty Publications by an authorized administrator of BYU ScholarsArchive. For more information, please contact ellen_amatangelo@byu.edu. 


\title{
Theory of an atomic beam splitter based on velocity-tuned resonances
}

\author{
S. Glasgow \\ Program in Applied Mathematics, University of Arizona, Tucson, Arizona 85721 \\ P. Meystre, M. Wilkens, and E. M. Wright* \\ Optical Sciences Center, University of Arizona, Tucson, Arizona 85721
}

(Received 15 October 1990)

\begin{abstract}
We develop the theory of an atomic beam splitter in which a monoenergetic beam of two-level atoms is incident normally to a classical standing-wave light field. The incident atomic wave function can be split into two coherent components with transverse momenta $\pm(2 n+1) \hbar k$ using velocity-tuned resonances, where $n$ is the order of the resonance. We discuss the cases of zero- and first-order resonances in detail, and show that the velocity-tuned resonances are renormalized due to a high-frequency Stark shift. Numerical results that display the effects of a finite momentum spread in the incident atomic beam are presented.
\end{abstract}

\section{INTRODUCTION}

There has been considerable recent interest in developing matter-wave interferometers employing neutral atoms. Potential applications include ultrahigh sensitivity accelerometers and gyroscopes as well as highresolution spatial spectroscopy. ${ }^{1}$ One of the major challenges in the design of such interferometers is to obtain large enough scattering angles that the atomic wave function becomes truly spatially separated. In simple-minded atomic beam splitters using, say, the near-resonant Kapitza-Dirac effect, ${ }^{2}$ the scattering angle is of the order of $\hbar k / m v$, where $k$ is the wave number of the light, $m$ the atomic mass, and $v$ its velocity. For typical atomic velocities of the order of hundreds of meters per second and visible light, this corresponds to very small angles, indeed. There are, however, a number of ways out of this difficulty, the most obvious one being to reduce the atomic velocity. Another recently proposed technique uses multiple-beam interactions with three-level atoms. ${ }^{3}$ In the so-called Bragg regime of atomic diffraction, strict energy-momentum conservation limits the scattering orders to a few well-determined values. Specifically, if the atoms enter the electromagnetic field with a transverse momentum $m \hbar k$, they are effectively coupled only to the state with transverse momentum $-m \hbar k$, and the evolution between these two states is governed by a Pendellösung-type oscillation. ${ }^{4-6}$ However, the frequency of oscillation of these solutions scales as $(\Omega / \Delta \omega)^{2 m-1}$, where $\Omega$ is the Rabi frequency and $\Delta \omega$ the atom-field detuning, making this method impractical since one needs to have $\Delta \omega \gg>$ to minimize the effects of spontaneous emission. In this paper, we discuss an alternative method based on the concept of Dopplerons, or velocity-tuned resonances. 7,8 In Doppleron resonances, the field frequency is chosen such that moving atoms use Doppler shifts to satisfy energy conservation in multiphoton transitions between electronic states. We show that this technique presents the advantage of leading to an effective separation of the atomic wave function over time scales considerably shorter than those involved in Pendellösung-type scattering. However, it also suffers from a number of difficulties, especially for high-order Dopplerons. In particular, the atom-field detuning must be chosen very accurately. For realistic atomic wave functions with a finite beam profile, this can lead to a severe breakup of the scattered wave function.

This paper is organized as follows: Section II presents the basic model used to describe the interaction between a two-level atom and a classical standing wave, and develops the equations of motion for the atomic probability amplitudes in momentum space. It then reviews the kinematic argument first advanced by Pritchard and Gould ${ }^{8}$ to determine the location of the Doppleron resonances. Section III considers the situation of an atomic beam splitter, using both zeroth-order and higher-order Dopplerons. The kinematic resonance condition is corrected to account for a Stark shift resulting from interactions with nonresonant electrotranslational levels. These corrections are shown to be negligible for zerothorder Dopplerons, but become essential in higher-order cases, where they imply stringent constraints on the atom-field detuning. So far, the discussion is concerned with plane atomic wave functions. Section IV relaxes this limitation, and shows how the narrow resonance condition derived in Sec. III can lead to a breakup of the atomic wave function for atomic beams of finite extent. Finally, Sec. V is a summary and conclusion.

\section{BASIC MODEL AND THEORY}

In this section we give the basic model used to describe an atomic beam splitter using Doppleron resonances. The pertinent equations are developed in Sec. II A, and a kinematic picture of the Doppleron resonance condition is given in Sec. II B. 


\section{A. Model and equations}

The atomic beam splitter under consideration is illustrated in Fig. 1. A monoenergetic beam of two-level atoms of mass $m$, electronic spacing $\hbar \omega_{0}$, and traveling at velocity $v_{z}$ along the $z$ axis is incident normally to a monochromatic classical standing-wave light field of frequency $\omega$. The electric field is polarized in the transverse $y$ direction. In the rotating-wave approximation and in the coordinate representation, the atomic evolution is adequately described by the pair of coupled Schrödinger equations, ${ }^{9}$

$$
i \hbar \frac{\partial a}{\partial t}=-\frac{\hbar^{2}}{2 m} \frac{\partial^{2} a}{\partial x^{2}}+\frac{\hbar \Omega}{2} \cos (k x) b
$$

and

$$
i \hbar \frac{\partial b}{\partial t}=-\frac{\hbar^{2}}{2 m} \frac{\partial^{2} b}{\partial x^{2}}-\hbar \Delta \omega b+\frac{\hbar \Omega}{2} \cos (k x) a,
$$

where $a(x, t)$ and $b(x, t)$ are the wave functions for the upper and lower electronic states of the two-level atoms, $\Delta \omega=\omega_{0}-\omega$ is the atom-field detuning, $k=\omega / c$ is the light wave vector, and $\Omega$ is the field Rabi frequency. The time variable $t$ appearing in Eqs. (1) is the retarded time in a reference frame moving at velocity $v_{z}$ along the $z$ axis. Physically, this implies that $v_{z}$ is assumed to be large enough that it can be described as a classical variable, and that the light field turns on "abruptly" at $t=0$. By "abruptly," we mean sufficiently fast to take $\Omega$ as a constant in Eqs. (1), but also slowly enough for the effects of the gradient force in the $z$ direction to be negligible. We also ignore the effects of spontaneous emission from the upper to lower electronic level.

It is convenient to consider input atomic wave functions corresponding to transverse momentum eigenstates. This allows us to expand the upper and lower state wave functions as spatial Fourier series over transverse momentum eigenstates 9,10

$$
a(x, t)=\sum_{j=-\infty}^{\infty} a_{j}(t) e^{i j k x}, \quad b(x, t)=\sum_{j=-\infty}^{\infty} b_{j}(t) e^{i j k x} .
$$

Such an expression is particularly appropriate to describe

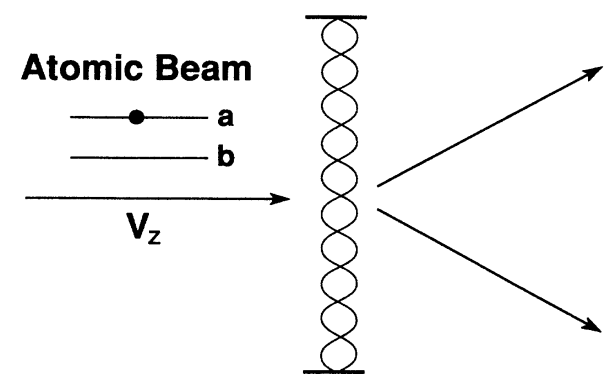

FIG. 1. Geometry of the proposed atomic beam splitter. The atoms enter the standing-wave light field in their excited state $|a\rangle$ and have longitudinal velocity $v_{z}$. the situation where the atoms are injected in their upper electronic state and with no transverse momentum, $j=0$ i.e., for the initial conditions

$$
a_{j}(0)=\delta_{j, 0}, \quad b_{j}(0)=0 .
$$

In this case, the atom-field interaction can only produce new scattering orders (transverse momentum eigenstates) corresponding to integer multiples of $\hbar k$, the quantum of transverse momentum exchanged between the atom and the field in elementary absorption and emission processes, and the expansions given by Eqs. (2) are valid. Then, for example, $\left|a_{j}(t)\right|^{2}$ is the probability of finding the atom in its upper state with transverse momentum $j \hbar k$ at time $t$.

Substituting the expansions of Eqs. (2) into the Schrödinger equations (1) yields equations for the timedependent amplitudes $a_{j}(t)$ and $b_{j}(t)$. For the initial conditions (3), we have the symmetries

$$
a_{j}(t)=a_{-j}(t), \quad b_{j}(t)=b_{-j}(t),
$$

with $j=1,2,3, \ldots$. Furthermore, the various amplitudes couple according to the scheme shown in Fig. 2(a). That is, for example, $a_{2}$ couples directly to $b_{1}$ and $b_{3}$ only. Thus we need only consider even values of $j$ for $a_{j}$, and odd values of $j$ for $b_{j} .{ }^{9}$ By making use of these properties, we obtain the system of equations

$$
\begin{aligned}
& i \frac{d a_{0}}{d t}=\frac{\Omega}{2} b_{1}, \\
& i \frac{d a_{j}}{d t}=j^{2} \omega_{R} a_{j}+\frac{\Omega}{4}\left(b_{j-1}+b_{j+1}\right), j=2,4,6 \\
& i \frac{d b_{i}}{d t}=\left(j^{2} \omega_{R}-\Delta \omega\right) b_{j}+\frac{\Omega}{4}\left(a_{j-1}+a_{j+1}\right), j=1,3,5, \ldots,
\end{aligned}
$$

where $\omega_{R}=\hbar k^{2} / 2 m$ is the recoil frequency. Note that,
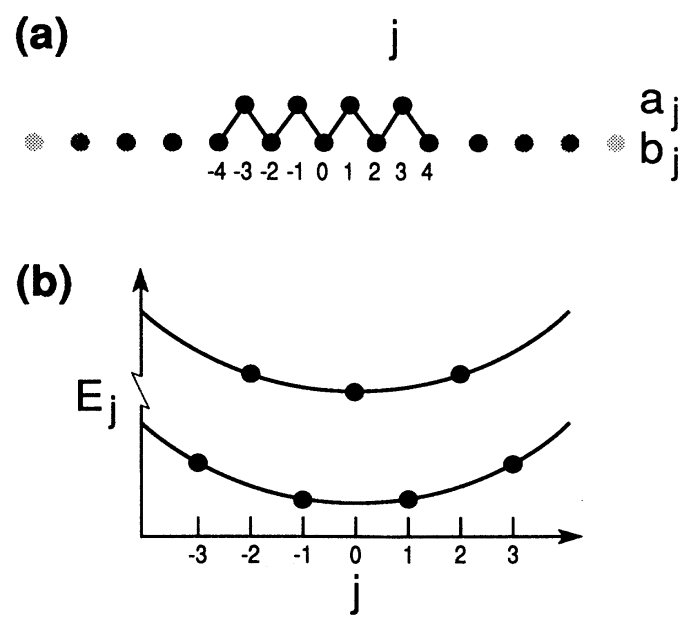

FIG. 2. (a) Coupling scheme between the excited- and ground-state amplitudes $a_{j}$ and $b_{j}$. (b) Bare energies $E_{j}$ of the various states of the system. In both of these figures $j$ even corresponds to the excited state of the atom and $j$ odd to the ground state. 
in comparison to Eqs. (5b) and (5c), the right-hand side of (5a) involves a factor $\Omega / 2$ as opposed to $\Omega / 4$. This is because $a_{0}$ interacts with both $b_{1}$ and $b_{-1}$ and these amplitudes are equal according to Eqs. (4).

In this paper, we concentrate on the use of Doppleron resonances to operate an atomic beam splitter. The concept of Dopplerons, or velocity-tuned resonances was originally introduced by Kyrölä and Stenholm, ${ }^{7}$ who considered situations in which the atoms have an initial transverse momentum satisfying $\left|p_{x}\right|>>\hbar k$, so that the atomic velocity remains essentially unchanged in absorption and emission processes. This approximation amounts to neglecting the effect of atomic recoil and effectively dropping the kinetic energy terms in Eqs. (1). Kyrölä and Stenholm did, however, retain the first-order Doppler effect by employing a convective derivative $\left[\partial / \partial t+\left(p_{x} / m\right) \partial / \partial x\right]$ in place of the time derivative in Eqs. (1). In contrast, our work assumes that the atom initially has zero transverse momentum, and it is therefore essential to retain both the first-order Doppler effect and atomic recoil, as described fully by the kinetic energy terms in Eqs. (1). In other words, we have to consider the full electrotranslational states of the atom instead of electronic states only.

\section{B. Kinematic picture}

The solutions of Eqs. (5) have been studied in detail on resonance by several authors, both theoretically ${ }^{9-14}$ and experimentally. ${ }^{15-20}$ In the limit that the recoil frequency is negligible compared to the Rabi frequency, the solutions are given in terms of Bessel functions, and the spread in atomic transverse momentum increases linearly with time. ${ }^{9}$ Similar results are found in the highly detuned case $|\Delta \omega / \Omega| \gg 1 .^{17}$ However, when the effects of atomic recoil are retained the growth in the spread in atomic transverse momentum becomes bounded. ${ }^{9,10}$ In particular, when the atomic recoil frequency becomes of the order of the single-photon Rabi frequency (we shall quantify these statements in the following sections) it becomes possible to limit the atom scattering to only a few scattering orders. In this sense atomic recoil can be seen as a phase-matching constraint which limits the proliferation of scattering orders.

The other parameter of central importance to our discussion is the atom-field detuning $\Delta \omega$, as it can be used to control the occurrence of certain types of atomic processes over others. On resonance, single-photon processes dominate, and the scattering orders correspond to transverse momenta which are integer multiples of $\hbar k$. Far off-resonance, in contrast, only virtual transitions between the upper and lower electronic states take place, and the scattering orders corresponding to transverse momenta which are integer multiples of $2 \hbar k$, dominate. In general, if an $m$-photon process is dominant, the important scattered components are at integer multiples of $m \hbar k$. However, if atomic recoil is effective, then it is possible to limit the scattered components to $\pm m \hbar k$.

In this paper, we consider specifically $n$ th-order Doppleron resonances, which correspond to atom-field interactions for which $(2 n+1)$ elementary emission or ab- sorption processes occur. For atoms initially in their upper state, the scattered components at $\pm(2 n+1) \hbar k$ are therefore necessarily in their lower state. With the above discussion in mind, a kinematic picture of the conditions under which Doppleron resonances occur can be obtained by using the simple model discussed by Pritchard and Gould. ${ }^{8}$ The bare energies (without the atom-field interaction energy) of the various states of the system can be written as

$$
E_{j}=j^{2} \hbar \omega_{R}+\left[1+(-1)^{j}\right] \hbar \omega_{0} / 2,
$$

where $j$ even (odd) corresponds to the upper (lower) state. These states are illustrated schematically in Fig. 2(b). The $n$ th-order Doppleron resonance occurs when

$$
E_{0}-E_{2 n+1}=\hbar \omega
$$

or for the atom-field detuning

$$
\Delta \omega_{n}^{(0)}=(2 n+1)^{2} \omega_{R},
$$

where the subscript $n$ labels the $n$ th-order Doppleron resonance. The reason for the superscript will become clear in the following sections. If this condition is satisfied, and assuming that the atomic recoil is sufficient to constrain the scattering orders, the initial atomic wave function will then be split into two coherent components corresponding to transverse momentum eigenstates with momenta $\pm(2 n+1) \hbar k$. Finally, for future use we define the effective detuning from one-photon resonance for the $n$ th-order Doppleron resonance as ${ }^{8}$

$$
\begin{aligned}
\delta_{n}=\left(E_{0}-E_{1}\right) \hbar-\omega & =\Delta \omega_{n}^{(0)}-\omega_{R} \\
& =\left[(2 n+1)^{2}-1\right] \omega_{R} .
\end{aligned}
$$

\section{ATOMIC BEAM SPLITTER}

In this section we analyze atomic beam splitters using both (a) zeroth-order, and (b) first-order Doppleron resonances. We comment on the use of still higher-order Doppleron resonances in Sec. III C.

\section{A. Zeroth-order Doppleron resonance}

In this case the atomic wave function, which is initially given by Eq. (3), is to be split into two coherent components, both corresponding to the atom being in its lower elextronic state, and with transverse momenta $\pm \hbar k$. To analyze this situation we truncate the equations of motion (5) beyond $j \geq 3$, which yields

$$
\begin{aligned}
& i \frac{d a_{0}}{d t}=\frac{\Omega}{2} b_{1}, \\
& i \frac{d b_{1}}{d t}=\left(\omega_{R}-\Delta \omega\right) b_{1}+\frac{\Omega}{4}\left(a_{0}+a_{2}\right), \\
& i \frac{d a_{2}}{d t}=4 \omega_{R} a_{2}+\frac{\Omega}{4} b_{1} .
\end{aligned}
$$

Note that we need to retain the amplitude $a_{2}$ since it is coupled directly to $b_{1}$. According to Eq. (8), the atomfield detuning for the zeroth-order Doppleron resonance 
is $\Delta \omega_{0}^{(0)}=\omega_{R}$. Under this condition we see from Eqs. (10a) and (10b) that the amplitudes $a_{0}$ and $b_{1}$ are phase synchronous, whereas $a_{2}$ is clearly phase mismatched. It then follows that $a_{2}$ is nonresonant and can be adiabatically eliminated by setting $d a_{2} / d t=0$ and solving for $a_{2}$. Equation (10b) then becomes

$$
i \frac{d b_{1}}{d t}=\left(\omega_{R}-\Omega^{2} / 64 \omega_{R}-\Delta \omega\right) b_{1}+\frac{\Omega}{4} a_{0} .
$$

This equation gives a corrected value for the resonance condition of the zeroth-order Doppleron resonance as

$$
\Delta \omega_{0}=\Delta \omega_{0}^{(0)}+\Delta \omega_{0}^{(1)}=\omega_{R}\left[1-\left(\Omega / 8 \omega_{R}\right)^{2}\right] .
$$

The first-order correction $\Delta \omega_{0}^{(1)}=-\omega_{R}\left(\Omega / 8 \omega_{R}\right)^{2}$ arises from the coupling of $b_{1}$ to $a_{2}$, and can therefore be seen as a renormalization of the resonance condition predicted from the kinematic picture. In the field of highresolution spectroscopy the shift of a resonance due to interaction with nonresonant levels is referred to as a highfrequency Stark shift. ${ }^{21,22}$ For the case considered here the level $a_{2}$ is nonresonant due to the effects of atomic recoil. We therefore interpret the shift in the zerothorder Doppleron resonance condition as a Stark shift due to coupling to neighboring electrotranslational states. The theory will be valid if this first-order correction is small, which requires

$$
\Omega / 8 \omega_{R}<1 .
$$

This condition also guarantees that the higher amplitudes with $j \geq 2$ do not become significantly excited.

Under conditions of zeroth-order resonance Eqs. (10a) and (11) can easily be solved for $a_{0}(t)$ and $b_{1}(t)$. We can then obtain expressions for $\left|a_{0}(t)\right|^{2}$ and $\left|b_{1}(t)\right|^{2}$ $=\left|b_{-1}(t)\right|^{2}$, which are the probabilities for finding the atom with transverse momenta $0, \pm 1$. A simple calculation yields

$$
\left|a_{0}(t)\right|^{2}=\cos ^{2}\left(\Omega_{01} t / 2\right),
$$

and

$$
\left|b_{1}(t)\right|^{2}=\left|b_{-1}(t)\right|^{2}=\frac{1}{2} \sin ^{2}\left(\Omega_{01} t / 2\right),
$$

where the effective Rabi frequency for coupling from $a_{0}$ to $b_{1}$ (or $b_{-1}$ ) is $\Omega_{01}=\Omega / \sqrt{2}$. This implies that the effective field causing transitions from $a_{0}$ to $b_{ \pm 1}$ is $1 / \sqrt{2}$ of the applied field. For an interaction time $t_{\text {int }}=\pi / \Omega_{01}$ the incident atomic wave function splits into two coherent components with transverse momenta $\pm \hbar k$.

To corroborate these predictions we have solved the full set of coupled equations (5) with up to 20 scattering orders. The results are shown in Fig. 3 where the probabilities $P_{j}=\left|a_{j}\right|^{2}, j$ even, and $P_{j}=\left|b_{j}(t)\right|^{2}, j$ odd, for finding the atom in the various scattering orders are plotted as functions of $\Omega t / 2$. The detuning was chosen as that for the zeroth-order Doppleron resonance, Eq. (12), and results were obtained for various values of $\Omega / 8 \omega_{R}$, which according to Eq. (13) should be much less than 1 . As shown in Fig. 3(a) and 3(b), the probability $P_{2}=P_{-2}$ of finding an atom with a transverse momentum $\pm 2 \hbar k$ can exceed $10 \%$ for $\Omega / 8 \omega_{R}>\frac{1}{2}$. In particular, for
$\Omega / 8 \omega_{R}=1$ [Fig. 3(a)] it is clearly seen that the higher scattering orders are being resonantly excited. In contrast, for $\Omega / 8 \omega_{R} \leq \frac{1}{4}, P_{2}$ becomes less than $1 \%$, and the zeroth-order Doppleron resonance can be used as an effective atomic beam splitter.

The width of the zeroth-order Doppleron resonance can be obtained from Eqs. (10a) and (11) by setting $\Delta \omega=\Delta \omega_{0}+\delta \omega$, where $\delta \omega$ is the detuning from resonance. By solving these equations as a function of $\delta \omega$ one finds that the zeroth-order resonance has a width $\delta \omega \simeq \Omega_{01}$. Note that the first-order correction $\left|\Delta \omega_{0}^{(1)}\right|$ $\simeq\left(\Omega / 8 \omega_{R}\right) \Omega_{01}$ is considerably smaller than the band-

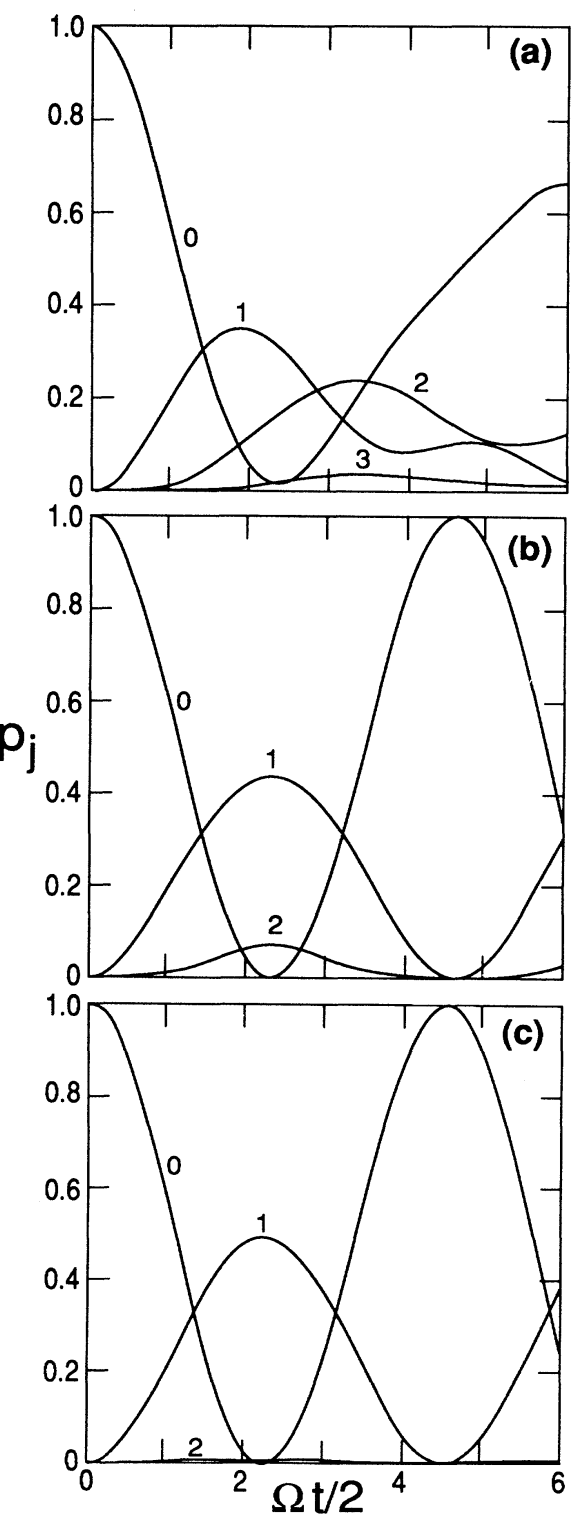

FIG. 3. Probabilities $P_{j}$ of finding the atom with transverse momentum $j \hbar k$ as a function of the dimensionless time $\Omega t / 2$, under conditions of zeroth-order Doppleron resonance and (a) $\Omega / 8 \omega_{R}=1$, (b) $\Omega / 8 \omega_{R}=\frac{1}{2}$, and (c) $\Omega / 8 \omega_{R}=\frac{1}{4}$. The value of $j$ is displayed beside each curve. 
width $\delta \omega$, since we require that $\Omega / 8 \omega_{R} \leq \frac{1}{4}$ for an effective atomic beam splitter. Therefore, the results shown in Fig. 3 are essentially the same whether or not we include the renormalization due to the high-frequency Stark shift. In the following sections we shall see that this is not the case for higher-order Dopplerons.

\section{B. First-order Doppleron resonance}

For the case of the first-order Doppleron resonance the incident atomic beam wave function can be split into two coherent parts of transverse momenta $\pm 3 \hbar k$. To analyze this case we truncate Eqs. (5) beyond $j \geq 5$, which yields

$$
\begin{aligned}
& i \frac{d a_{0}}{d t}=\frac{\Omega}{2} b_{1}, \\
& i \frac{d b_{1}}{d t}=\left(\omega_{R}-\Delta \omega\right) b_{1}+\frac{\Omega}{4}\left(a_{0}+a_{2}\right), \\
& i \frac{d a_{2}}{d t}=4 \omega_{R} a_{2}+\frac{\Omega}{4}\left(b_{1}+b_{3}\right), \\
& i \frac{d b_{3}}{d t}=\left(9 \omega_{R}-\Delta \omega\right) b_{3}+\frac{\Omega}{4}\left(a_{2}+a_{4}\right), \\
& i \frac{d a_{4}}{d t}=16 \omega_{R} a_{4}+\frac{\Omega}{4} b_{3} .
\end{aligned}
$$

We retain the amplitude $a_{4}$ since it is coupled directly to $b_{3}$. Equation (8) gives the resonance condition for the first-order Doppleron $\Delta \omega_{1}^{(0)}=9 \omega_{R}$. Under this condition we see from Eqs. (15a) and (15d) that the amplitudes $a_{0}$ and $b_{3}$ are phase synchronous, whereas the remaining amplitudes $b_{1}, a_{2}$, and $a_{4}$ are phase mismatched. We therefore adiabatically eliminate these amplitudes using $\Delta \omega=\Delta \omega_{1}^{(0)}$ in Eq. (15b), which yields

$$
\left[\begin{array}{cc}
8 \omega_{R} & -\Omega / 4 \\
-\Omega / 4 & -4 \omega_{R}
\end{array}\right]\left[\begin{array}{l}
b_{1} \\
a_{2}
\end{array}\right]=\frac{\Omega}{4}\left[\begin{array}{l}
a_{0} \\
b_{3}
\end{array}\right)
$$

and

$$
a_{4}=-\left(\frac{\Omega}{64 \omega_{R}}\right) b_{3} .
$$

Note that from Eq. (17) the magnitude of $a_{4}$ is of order $\epsilon=\Omega / \omega_{R}$. Equation (16) can easily be inverted to yield $b_{1}$ and $a_{2}$ as functions of $a_{0}$ and $b_{3}$. If we perform this inversion and retain only terms up to order $\epsilon^{2}$ we obtain

$$
\left[\begin{array}{l}
b_{1} \\
a_{2}
\end{array}\right]=\left(\begin{array}{cc}
\Omega / 32 \omega_{R} & -\Omega^{2} / 512 \omega_{R}^{2} \\
-\Omega^{2} / 512 \omega_{R}^{2} & -\Omega / 16 \omega_{R}
\end{array}\right]\left(\begin{array}{l}
a_{0} \\
b_{3}
\end{array}\right) .
$$

Substituting Eqs. (17) and (18) into Eqs. (15a) and (15d), and setting $\Delta \omega=\Delta \omega_{1}^{(0)}+\Delta \omega_{1}^{(1)}$ in Eq. (15d), yields

$$
\begin{aligned}
& i \frac{d a_{0}}{d t}=\frac{1}{64}\left(\frac{\Omega^{2}}{\omega_{R}}\right) a_{0}-\frac{1}{1024}\left(\frac{\Omega^{3}}{\omega_{R}^{2}}\right) b_{3}, \\
& i \frac{d b_{3}}{d t}=-\left[\Delta \omega_{1}^{(1)}+\frac{5}{256}\left(\frac{\Omega^{2}}{\omega_{R}}\right)\right] b_{3}-\frac{1}{2048}\left(\frac{\Omega^{3}}{\omega_{R}^{2}}\right) a_{0} .
\end{aligned}
$$

In order that $a_{0}$ and $b_{3}$ be phase synchronous, we require that the diagonal terms in Eqs. (19) be equal. This yields the first-order Doppleron resonance condition

$$
\Delta \omega_{1}=\Delta \omega_{1}^{(0)}+\Delta \omega_{1}^{(1)}=9 \omega_{r}\left[1-\left(\Omega / 16 \omega_{R}\right)^{2}\right] .
$$

Therefore, as with the zeroth-order resonance, the resonance condition is renormalized due to the coupling of the amplitudes of interest, $a_{0}$ and $b_{3}$, with the nonresonant amplitudes. Again, this can be interpreted as a high-frequency Stark shift due to nonresonant electrotranslational states. However, in contrast to the zerothorder Doppleron, where we have seen that the correction $\Delta \omega_{0}^{(1)}$ is of little significance, for the first-order Doppleron the inclusion of the renormalization is of central importance, as shall be seen below. Note that our analysis is only valid if this correction term is small, which requires

$$
\Omega / 16 \omega_{R}<1 .
$$

This condition also guarantees that the $b_{1}, a_{2}$, and higher amplitudes with $j \geq 4$ do not become significantly excited.

Under conditions of first-order Doppleron resonance Eqs. (19) can easily be solved for $a_{0}(t)$ and $b_{3}(t)$ to give

$$
\left|a_{0}(t)\right|^{2}=\cos ^{2}\left(\Omega_{03} t / 2\right)
$$

and

$$
\left|b_{3}(t)\right|^{2}=\left|b_{-3}(t)\right|^{2}=\frac{1}{2} \sin ^{2}\left(\Omega_{03} t / 2\right),
$$

where the effective three-photon Rabi frequency for coupling from $a_{0}$ to $b_{3}$ (or $b_{-3}$ ) is given by

$\Omega_{03}=\frac{1}{2 \sqrt{2}}\left(\frac{\Omega}{16 \omega_{R}}\right)^{2} \Omega=\left(\frac{\Omega / \sqrt{2}}{2 \delta_{1}}\right)^{2}(\Omega / \sqrt{2})$.

Here, following the notation introduced in Sec. II B, we have used the effective detuning from one-photon resonance $\delta_{1}=8 \omega_{R}$. Taking account of the fact that the effective field is $1 / \sqrt{2}$ of the applied field (see Sec. III A), Eq. (23) is the standard result for the effective threephoton Rabi frequency. 8,21 Therefore, for an interaction time $t_{\text {int }}=\pi / \Omega_{03}$ the incident atomic wave function is split into two coherent components with transverse momenta $\pm 3 \hbar k$.

Figure 4 shows numerical results for the probabilities $P_{j}$ of finding the atom in the various scattering orders as functions of $\Omega t / 2$. In Figs. 4(a) and 4(b) the detuning was chosen as that for the first-order Doppleron resonance, Eq. (20), and the values of $\Omega / 8 \omega_{R}$ used were (a) $\frac{1}{2}$ and (b) $\frac{1}{4}$. As shown in Fig. 4(a) the probabilities $P_{1,2}$ of finding an atom with transverse momentum $\pm \hbar k$, and $\pm 2 \hbar k$ can exceed $20 \%$ for $\Omega / 16 \omega_{R}>\frac{1}{2}$. In contrast, for $\Omega / 16 \omega_{R} \leq \frac{1}{4}$ [Fig. 4(b)] $P_{1,2}$ become less than $1 \%$, and the first-order Doppleron resonance can be used as an effective atomic beam splitter. Note, however, the difference in time scales in Figs. 4(a) and 4(b). It follows from $\mathrm{Eq}$ (23) that, for a fixed Rabi frequency $\Omega$, the interaction time $t_{\text {int }}=\pi / \Omega_{03}$ required for an atomic beam splitter scales as $\left(\Omega / 16 \omega_{R}\right)^{-2} \simeq(9 \Omega / 16 \Delta \omega)^{-2}$, where we have used the zeroth-order Doppleron resonance condition. This scaling is much more favorable than for an 


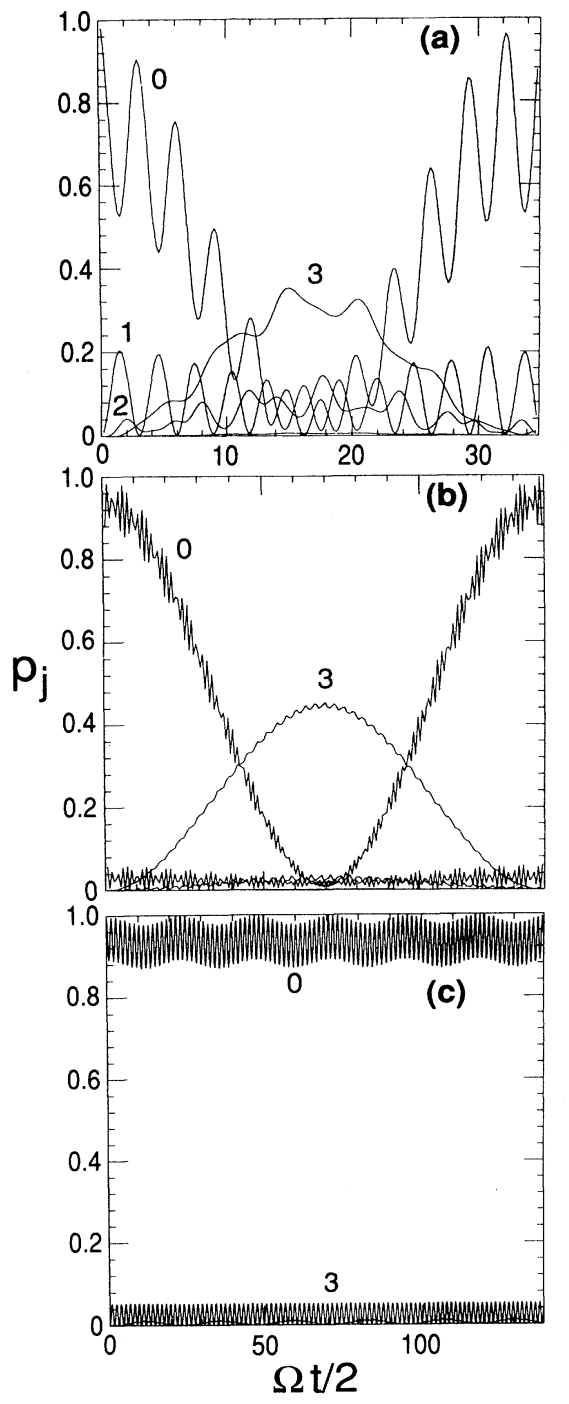

FIG. 4. Probabilities $P_{j}$ of finding the atom with transverse momentum $j \hbar k$ as a function of the dimensionless time $\Omega t / 2$, under conditions of first-order Doppleron resonance and (a) $\Omega / 8 \omega_{R}=\frac{1}{2}$ and (b) $\Omega / 8 \omega_{R}=\frac{1}{4}$. (c) was also obtained using $\Omega / 8 \omega_{R}=\frac{1}{4}$ but without the first-order correction in Eq. (20). The value of $j$ is displayed beside each curve.

atomic beam splitter using Pendellösung oscillations between states of transverse momenta $3 \hbar k$ and $-3 \hbar k$, which is a six-photon process. The time scale for this process can be obtained by expanding the generalized Rabi frequency as

$$
\begin{aligned}
\Omega_{e} & =\left(\Omega^{2}+\Delta \omega^{2}\right)^{1 / 2} \\
& =\Delta \omega+\frac{\Omega^{2}}{2 \Delta \omega}-\frac{\Omega^{4}}{8 \Delta \omega^{3}}+\frac{\Omega^{6}}{16 \Delta \omega^{5}}-\cdots,
\end{aligned}
$$

where the fourth term corresponds to the six-photon process. Therefore, for a fixed Rabi frequency $\Omega$, the interaction time required for this process to operate as an atomic beam splitter scales as $(\Omega / 2 \Delta \omega)^{-5}$.
The width of the first-order Doppleron resonance can be obtained from Eqs. (19) and by setting $\Delta \omega=\Delta \omega_{1}+\delta \omega$, where $\delta \omega$ is the detuning from resonance. By solving these equations as a function of $\delta \omega$ one finds that it has a width $\delta \omega \simeq \Omega_{03}$. In this case, the first-order correction to the resonance frequency $\left|\Delta \omega_{1}^{(1)}\right| \simeq\left(\Omega / 16 \omega_{R}\right)^{-1} \Omega_{03}$, is larger than the bandwidth $\delta \omega$, since we require that $\Omega / 16 \omega_{R} \leq \frac{1}{4}$ for an effective atomic beam splitter. Therefore, for an atomic beam splitter based on a first-order Doppleron resonance, the renormalization of the resonant frequency due to the high-frequency Stark shift is of utmost importance. This is illustrated in Fig. 4(c), which corresponds to the same situation as shown in Fig. 4(b), but here we have set $\Delta \omega=\Delta \omega_{1}^{(0)}$, thus neglecting the first-order correction. In comparison to the resonant case shown in Fig. 4(b) we see that the system no longer acts as an atomic beam splitter.

\section{Higher-order Doppleron resonances}

It is possible to solve the system of equations (5) for higher-order Doppleron resonances $n \geq 1$. However, the adiabatic elimination procedure becomes more and more subtle due to the presence of an increasing number of detuned intermediate states. For example, for $n=2$ the resonance condition is given by

$$
\begin{aligned}
\Delta \omega_{2} & =\Delta \omega_{2}^{(0)}+\Delta \omega_{2}^{(1)}+\Delta \omega_{2}^{(2)} \\
& =\Delta \omega_{2}^{(0)}\left[1-\left(\Omega / 48 \omega_{R}\right)^{2}+\frac{5}{16}\left(\Omega / 48 \omega_{R}\right)^{4}\right],
\end{aligned}
$$

and the numerical calculations verify that it is important to retain the second-order correction in this expression.

We are currently developing a multiple-time-scale theory of an atomic beam splitter based on Doppleron resonances that reveals the properties of the system. However, some general properties are already apparent from the present work. First, it is clear that the renormalization of the resonance frequency becomes increasingly important for Dopplerons beyond first order. Second, the time scales necessary to operate the system as a beam splitter increase dramatically with increasing Doppleron order. This implies that a proper treatment of higher-order Doppleron resonances should incorporate the effects of spontaneous emission.

\section{FINITE ATOMIC BEAM EFFECTS}

In our treatment so far we have assumed that the input atomic wave function is a single transverse momentum eigenstate. In general, this will not be the case and the input is better described by a continuous superposition of transverse eigenstates. The variance in the transverse momentum of the input is then no longer zero, which implies, via the uncertainty principle, that we are dealing with atomic beams of finite transverse extent.

To treat such atomic beams of finite transverse extent we have solved Eqs. (1) numerically with the beam propagation method ${ }^{23}$ using the fast-Fourier-transform algorithm. We assume an initial Gaussian atomic wave function, 


$$
\begin{aligned}
& a(x, 0)=\left[2 \pi(\Delta x)^{2}\right]^{-1 / 4} \exp \left[-x^{2} / 4(\Delta x)^{2}\right], \\
& b(x, 0)=0,
\end{aligned}
$$

where $\Delta x$ is the rms fluctuation around $x=0$. From the uncertainty principle for a Gaussian wave packet we know that the fluctuations in transverse momentum $\Delta p$ are given by

$$
\Delta p=\hbar \Delta k=\hbar / 2 \Delta x,
$$

where $\Delta k$ is the rms fluctuation in the transverse wave vector. Numerically we solve for $a(x, t)$ and $b(x, t)$. We then convert this to momentum space by Fourier transformation to obtain $\bar{a}\left(p_{x}, t\right)$ and $\bar{b}\left(p_{x}, t\right)$. Then, for example, $\left|\bar{a}\left(p_{x}, t\right)\right|^{2}$ is the probability density for finding the atom in its excited state with transverse momentum $p_{x}$.

To obtain an idea of when finite beam effects are relevant it is possible to rework the calculations of Secs. II and III to incorporate an initial transverse momentum $p_{0}=\hbar k_{0}$. From these calculations one can obtain the bandwidth $\delta p=\hbar \delta k$ over which the initial atomic transverse momentum may deviate from zero before the Dop-

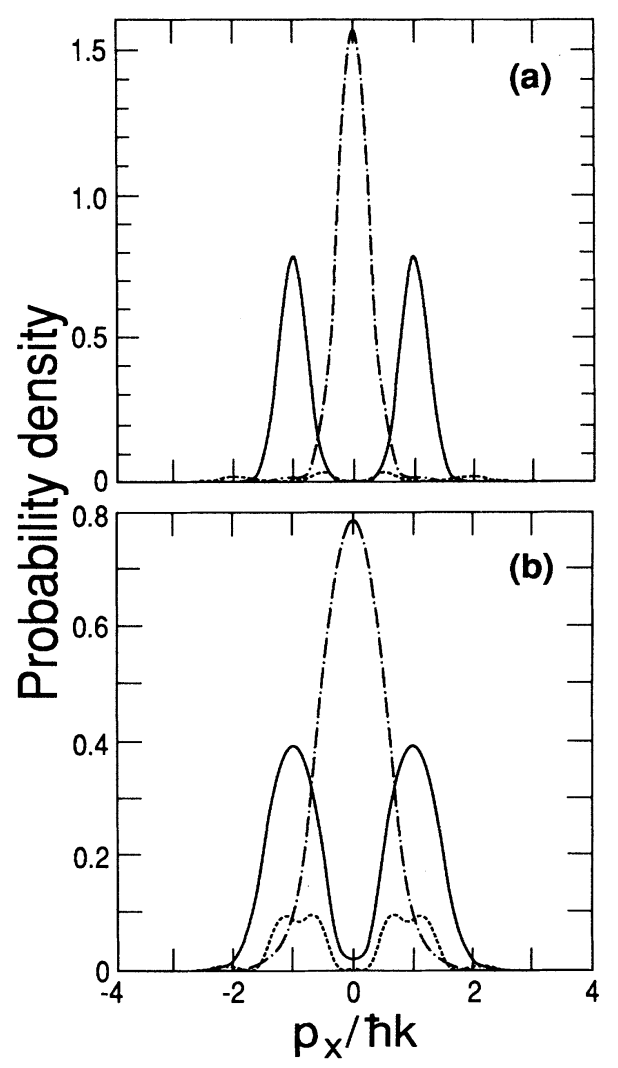

FIG. 5. Probability densities of finding the atom with scaled transverse momentum $p_{x} / \hbar k$, under conditions of zeroth-order Doppleron resonance for $\Omega / 8 \omega_{R}=\frac{1}{4}, \Omega t / 2=2.3$, and (a) $\Delta k / k=\frac{1}{4}$, (b) $\Delta k / k=\frac{1}{2}$. The dot-dashed line shows the initial excited-state probability density, and the solid and dashed lines show the final ground- and excited-state probability densities, respectively. pleron resonance is destroyed. For the cases of zerothorder and first-order Doppleron resonances we find

$$
\delta k_{0} \simeq\left(\Omega / 8 \omega_{R}\right) k
$$

and

$$
\delta k_{1} \simeq\left(\Omega / 16 \omega_{R}\right)^{3} k .
$$

Finite beam effects are relevant when $\Delta k$ is greater than the spatial frequency bandwidth $\delta k$.

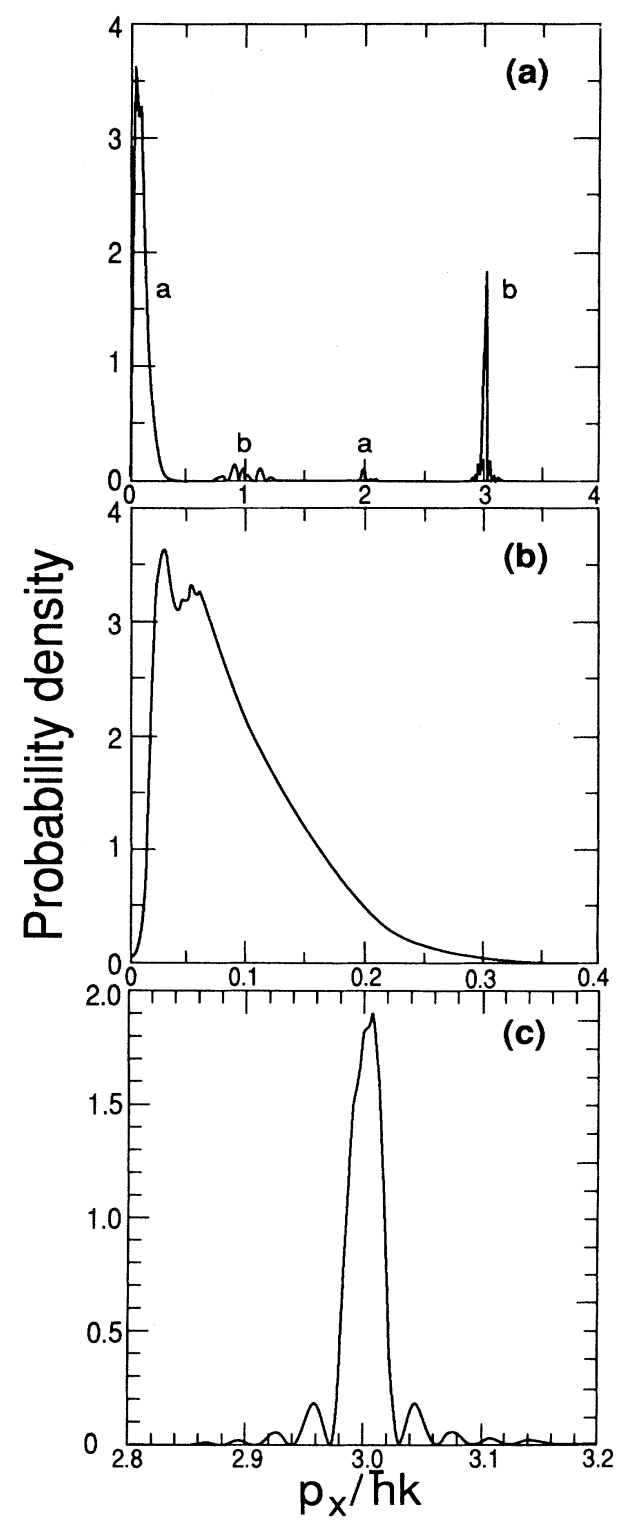

FIG. 6. Probability densities of finding the atom with scaled transverse momentum $p_{x} / \hbar k$, under conditions of first-order Doppleron resonance for $\Omega / 8 \omega_{R}=\frac{1}{4}, \Omega t / 2=70$, and (a) $\Delta k / k=\frac{1}{10}$, (b) enlargement of the region around $p_{x} / \hbar k=0$, and (c) enlargement of the region around $p_{x} / \hbar k=3$. The regions marked $a$ and $b$ correspond to the excited- and groundstate probability densities, respectively. 
Figure 5 shows $|\bar{a}|^{2}$ (dashed line), $|\bar{b}|^{2}$ (solid line), and the initial probability density (dash-dotted line) as functions of $p_{x} / \hbar k$ for the zeroth-order Doppleron resonance. The parameters used are the same as in Fig. 3(b), and for an interaction time $\Omega t_{\text {int }} / 2=2.3$. This is the time for which the system acts effectively as an atomic beam splitter [see Fig. 3(b)]. The two examples shown in Fig. 5 correspond to (a) $\Delta k / k=\frac{1}{4}$, and (b) $\Delta k / k=\frac{1}{2}$, whereas $\delta k_{0} / k=\frac{1}{2}$. For the case shown in Fig. 5(a), $\Delta k=\delta k / 2$, the input wave function is split as expected into two coherent components with mean transverse momenta $\pm \hbar k$. Note that there is relatively small probability of finding the atom in its excited state. In contrast, for the case shown in Fig. $5(\mathrm{~b}), \Delta k=2 \delta k$, and only the central portion of the incident atomic wave function is scattered by the Doppleron resonance, there now being a significant probability of finding the atom in its excited state. In this case the input spread in transverse momentum is larger than the spatial frequency bandwidth of the system, and this leads to breakup in the scattered wave functions.

Similar breakup phenomena also occur for high-order Dopplerons but are much more severe, as reflected in Eq. (28). Recalling that $\Omega / 16 \omega_{R}<\frac{1}{4}$ for the first-order Doppleron to be effective as an atomic beam splitter, this implies that the input spread in transverse atomic momentum must obey $\Delta k / k<0.02$ to avoid breakup. This is clearly a very stringent requirement on future experiments in this direction.

Figure 6(a) shows the probability densities $|\bar{a}|^{2}$ and $|\bar{b}|^{2}$ as functions of $p_{x} / \hbar k$ for the first-order Doppleron. The parameters used here are the same as in Fig. 4(b), and the interaction time is $\Omega t_{\text {int }} / 2=70$, for which the system acts effectively as an atomic beam splitter [see Fig. 4(b)]. The portions of the figure labeled (a) and (b) correspond to the excited- and ground-state wave functions, respectively. Here we have chosen $\Delta k / k=0.1$, whereas $\delta k / k=0.02$. Thus excessive breakup occurs in the scattered wave functions. This is illustrated in Figs. 6(b) and 6(c), where we show enlarged views of the regions around $p_{x}=0$ and $3 \hbar k$ from Fig. 6(a). Note in Fig. 6(b) that only the very central portion of the incident wave function is scattered by the Doppleron resonance. This is also evident in Fig. 6(c), where the scattered wave function has a width of around 0.02 , in excellent agreement with the predicted spatial frequency bandwidth.

\section{SUMMARY AND CONCLUSIONS}

In this paper, we have analyzed an atomic beam splitter using Doppleron, or velocity-tuned resonances. We have derived resonance conditions for $n$ th-order resonances, and shown that they differ from those derived from a simple kinematic argument by a correction that can be interpreted as a high-frequency Stark shift. For higher-order Doppleron resonances, the width of the resonance becomes extremely narrow, setting stringent requirements on acceptable initial momentum spreads. If these requirements are not met, the atomic wave function undergoes a severe breakup as the atom interacts with the electromagnetic field. Although it limits the practicality of using Doppleron resonances in an atom interferometer, this breakup can be understood as a spatial filtering of atomic momenta. As such, it represents a spatial counterpart of conventional high-resolution spectroscopy, and might also provide a basic element to prepare highly monochromatic atomic beams. These possibilities, as well as a full three-dimensional picture of the problem and an analysis of a complete atom interferometer, will be the subject of future work.

\section{ACKNOWLEDGMENTS}

This research is supported by the U. S. Office of Naval Research, Contract No. N00014-88-K-0294 and by the Joint Services Optics Program. Discussions with P. J. Martin are greatfully acknowledged.
*Also at Physics Department, University of Arizona, Tucson, AZ 85721.

${ }^{1}$ J. F. Clauser, Physica B 151, 262 (1988).

${ }^{2}$ P. L. Kapitza and P. A. M. Dirac, Proc. Cambridge Philos. Soc. 29, 297 (1933).

${ }^{3}$ P. J. Martin and J. Hall (unpublished).

${ }^{4}$ P. J. Martin, B. G. Oldaker, A. H. Miklich, and D. E. Pritchard, Phys. Rev. Lett. 60, 515 (1988).

${ }^{5}$ P. Meystre, E. Schumacher, and E. M. Wright, Ann. Phys. (Leipzig) (to be published).

${ }^{6}$ For an experimental verification of Pendellösung oscillations in neutron diffraction, see C. G. Shull, Phys. Rev. Lett. 21, 1585 (1968).

${ }^{7}$ E. Kyrölä and S. Stenholm, Opt. Commun. 22, 123 (1977); S. Stenholm, Foundations of Laser Spectroscopy (WileyInterscience, New York, 1984)

${ }^{8}$ D. E. Pritchard and P. L. Gould, J. Opt. Soc. Am. B 2, 1799 (1985).

${ }^{9}$ E. Arimondo, A. Bambini, and S. Stenholm, Optics Commun. 37, 103 (1981); Phys. Rev. A 24, 898 (1981).
${ }^{10}$ A. F. Bernhardt and B. W. Shore, Phys. Rev. A 23, 1290 (1981).

${ }^{11}$ R. J. Cook and A. F. Bernhardt, Phys. Rev. A 18, 2533 (1978).

${ }^{12}$ G. A. Delone, V. A. Grinchuk, S. D. Kuzmichev, M. L. Nagavea, A. P. Kasantsev, and G. I. Surdutovich, Opt. Commun. 33, 149 (1980).

${ }^{13}$ C. Tanguy, S. Reynaud, and C. Cohen-Tannoudji, J. Phys. B 17, 4623 (1984).

${ }^{14}$ A. P. Kazantsev, G. A. Ryabenko, G. I. Surdovich, and V. P. Yakovlev, Phys. Rep. 129, 77 (1985).

${ }^{15}$ E. Arimondo, H. Lew, and T. Oka, Phys. Rev. Lett. 43, 753 (1979).

${ }^{16}$ P. E. Moskowitz, P. L. Gould, S. R. Atlas, and D. E. Pritchard, Phys. Rev. Lett. 51, 370 (1983).

${ }^{17}$ P. L. Gould, G. A. Ruff, and D. E. Pritchard, Phys. Rev. Lett. 56, 827 (1986).

${ }^{18}$ C. Salomon, J. Dalibard, A. Aspect, H. Metcalf, and C. Cohen-Tannoudji, Phys. Rev. Lett. 59, 1659 (1987).

${ }^{19}$ P. J. Martin, P. L. Gould, B. G. Oldaker, A. H. Miklich, and D. E Pritchard, Phys. Rev. A 36, 2495 (1987); P. J. Martin, B. 
G. Oldaker, A. H. Miklich, and D. E. Pritchard, Phys. Rev. Lett. 60, 515 (1988).

${ }^{20}$ J. W. Early, Opt. Commun. 65, 250 (1988).

${ }^{21}$ T. Oka, in Frontiers in Laser Spectroscopy, edited by R. Balian, S. Haroche, and S. Liibermann (North-Holland, New York, 1977).
${ }^{22}$ See, e.g., P. Meystre and M. Sargent III, Elements of Quantum Optics (Springer-Verlag, Berlin 1990).

${ }^{23}$ J. W. Lewis and L. Solymar, Proc. R. Soc. London Ser. A 398, 45 (1985); R. V. Johnson and A. R. Tanguay, Opt. Eng. 25, 235 (1986). 